\title{
A Patient with Hematochezia and Intestinal Obstruction
}

\author{
Kyong Yong Oh, Yoon Jae Kim \\ Division of Gastroenterology, Department of Internal Medicine, Gachon University Gil Hospital, Incheon, Korea
}
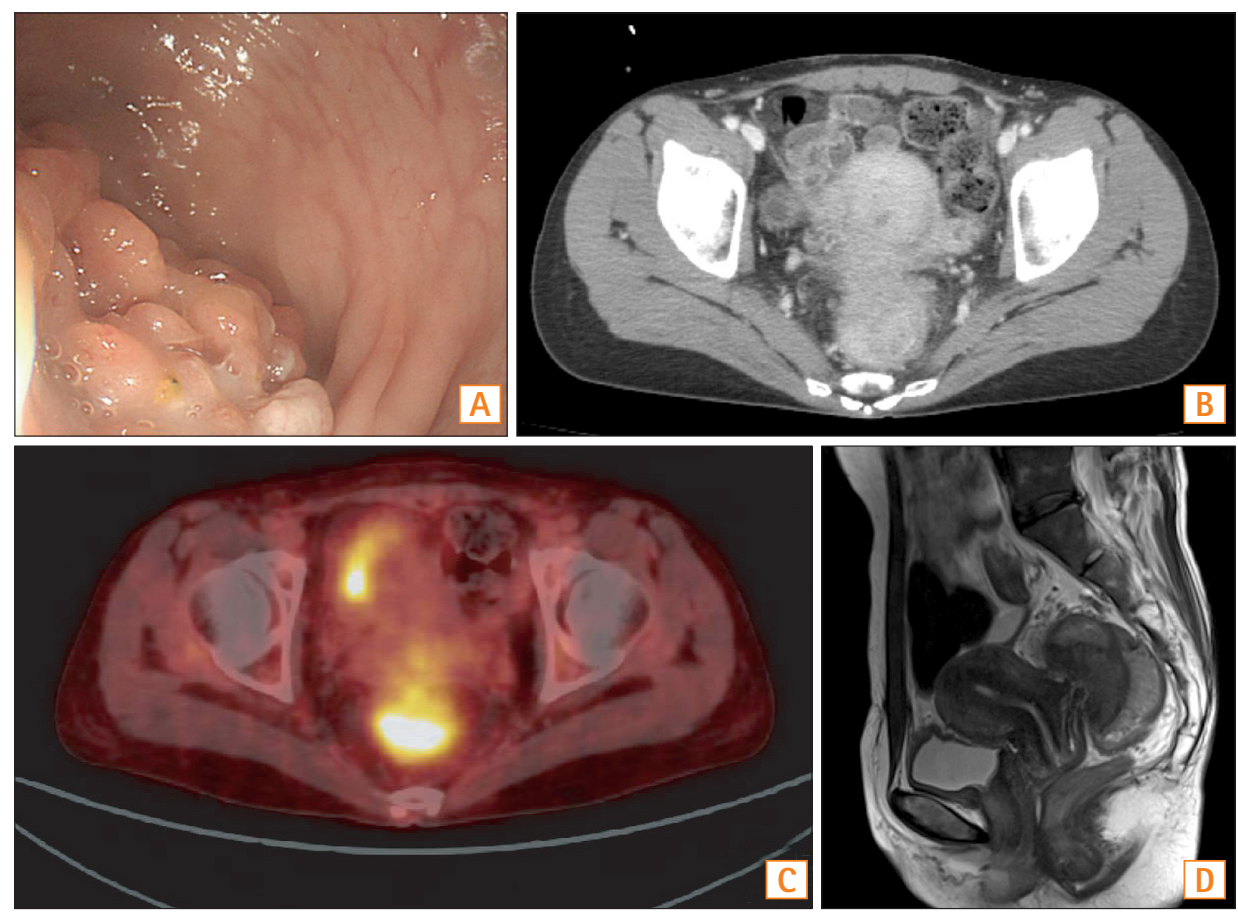

Question: A 37-year-old woman presented at our hospital with hematochezia (approximately $200 \mathrm{cc}$ ). She had experienced constipation and abdominal pain for one month, and four days previously had been admitted to the Department of Gynecology for a cervical polyp with vaginal bleeding. She had undergone bilateral salpingectomy for blocked fallopian tubes at another hospital 3 years prior to the current admission.

On admission, initial laboratory findings revealed anemia

Received June 3, 2014. Revised June 12, 2014. Accepted June 12, 2014. Correspondence to Yoon Jae Kim, Division of Gastroenterology, Department of Internal Medicine, Gachon University Gil Hospital, 24 Namdong-daero 774beon-gil, Namdong-gu, Incheon 405-760, Korea. Tel: +82-32-460-3778, Fax:+82-32-460-3408, E-mail: yoonmed@gachon.ac.kr

Financial support: None. Conflict of interest: None. (hemoglobin concentration, $7.4 \mathrm{~g} / \mathrm{dL}$ ), and elevated CA-125 levels $(75.10 \mathrm{U} / \mathrm{mL})$. Sigmoidoscopy was performed, showing a polypoid, fungating mass with colonic obstruction in the rectum, located $11 \mathrm{~cm}$ from the anal verge (Fig. A). Chronic inflammation with ulceration and epithelial hyperplasia were observed in the mucosal layer of endoscopic biopsies. A CT scan of the abdomen and pelvis revealed an illdefined enhancing lesion in the rectum, abutting the uterus (Fig. B). Strong fluorodeoxyglucose uptake in the rectum was noted on PET-CT scanning (Fig. C). Further analysis by T2W sagittal imaging and MRI confirmed the presence of a fungating rectal mass that was infiltrating the uterus (Fig. D).

Although the presence of cancer was not confirmed in colonoscopic biopsies, we decided to operate based on clinical suspicions of invasive rectal malignancy and colonic ob-

๑ Copyright 2014. Korean Association for the Study of Intestinal Diseases. All rights reserved.

This is an Open Access article distributed under the terms of the Creative Commons Attribution Non-Commercial License (http://creativecommons.org/licenses/by-nc/3.0)

which permits unrestricted non-commercial use, distribution, and reproduction in any medium, provided the original work is properly cited. 

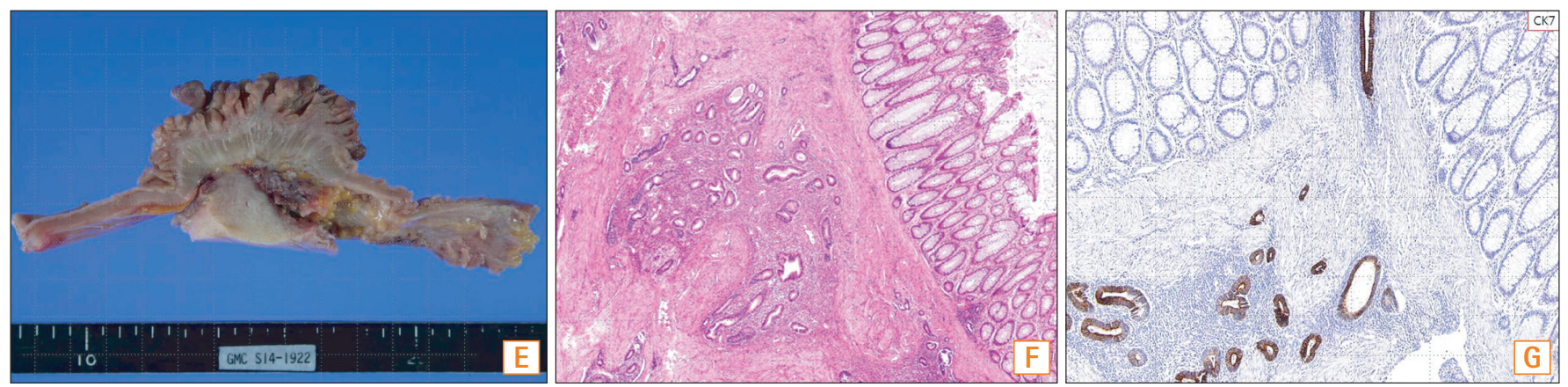

struction. The patient underwent low anterior resection and total abdominal hysterectomy. Numerous polypoid rectal masses were subsequently detected in the gross specimen. Severe fibrosis and adhesions between the rectum and the lateral wall of uterus were also observed (Fig. E). A pathologic analysis of H\&E staining $(\times 100)$ in the surgical specimen revealed an endometrial-like gland with multifocal stromal cells in the rectum and regional lymph node (Fig. F). Cytokeratin 7-positive cells were detected by immunohistochemical staining for cytokeratin 7 (Fig. G, $\times 100$ ).

What is the most likely diagnosis?

\section{Answer to the Images: Rectal Endometriosis Causing Colonic Obstruction}

Endometriosis of the bowel is indicated by the presence of an endometrial-like gland and stromal cells in the intestinal wall. The occurrence of bowel endometriosis in the general population is unknown, though it is estimated to afflict $3.8-37 \%$ of women with endometriosis. ${ }^{1}$ Intestinal endometriosis may affect the ileum, appendix, sigmoid colon and rectum, though it occurs most frequently in the rectosigmoid colon (50-90\%). ${ }^{1}$ Associated symptoms vary according to the site of involvement, as well as the size and depth of infiltration into the bowel wall. Symptoms usually include abdominal pain, bloating, nausea, vomiting, fecal tenesmus, painful defecation, alternating constipation and diarrhea, and rectal bleeding. ${ }^{2}$

Transvaginal ultrasound, CT scanning, MRI, and PET scanning are required to confirm diagnosis. ${ }^{3}$ Sigmoidoscopy and colonoscopy have limited value in the diagnosis of intestinal endometriosis, since it is rare for lesions to infiltrate the mucosa. ${ }^{1}$ It is important, however, to exclude the presence of colorectal cancer and to assess any bowel stenosis.

In the current case, we misdiagnosed intestinal endometriosis as colorectal cancer prior to surgery, based on the presence of hematochezia, and on sigmoidoscopic and radiologic findings, which were suggestive of invasive rectal malignancy. Moreover, sigmoidoscopic biopsies were inconclusive. A definitive diagnosis could only be made on the basis of pathologic analysis of surgical specimens. In women of reproductive age, therefore, clinical suspicion of bowel endometriosis is important in cases where an intestinal mass is detected with bleeding or intestinal obstruction.

\section{REFERENCES}

1. Remorgida V, Ferrero S, Fulcheri E, Ragni N, Martin DC. Bowel endometriosis: presentation, diagnosis, and treatment. Obstet Gynecol Surv 2007;62:461-470.

2. Shaw A, Lund JN, Semeraro D, Cartmill M, Reynolds JR, Tierney GM. Large bowel obstruction and perforation secondary to endometriosis complicated by a ventriculoperitoneal shunt. Colorectal Dis 2008;10:520-521.

3. Kim JS, Hur H, Min BS, et al. Intestinal endometriosis mimicking carcinoma of rectum and sigmoid colon: a report of five cases. Yonsei Med J 2009;50:732-735. 